\title{
Herbicide sequence for weed management in direct seeded rice
}

\author{
Y. M. Ramesha ${ }^{*}$, M.R. Umesh ${ }^{1}$, S. R. Anand ${ }^{1}$, Manjunatha Bhanuvally ${ }^{2}$ and Ashok \\ Kumar Gaddi ${ }^{2}$ \\ ${ }^{1}$ Department of Agronomy, University of Agricultural Sciences, Raichur, (Karnataka), INDIA \\ ${ }^{2}$ Department of Soil Science and Agricultural Chemistry, University of Agricultural Sciences, Raichur, \\ (Karnataka), INDIA \\ *Corresponding author. E-mail: rameshaym@gmail.com
}

Received: December 27, 2016; Revised received: March 16, 2017; Accepted: August 27, 2017

\begin{abstract}
An experiment was conducted during Kharif 2014 and 2015 at Agricultural Research Station, Dhadesugur, University of Agricultural Sciences, Raichur, Karnataka, India, to know the herbicide sequence for weed management in direct seeded rice. The dominant weeds in direct seeded rice were Echinochloa sp, Panicum repens, Cynodon doctylon, Leptochloa chinensis, Bracharia sp. Ludwigia parviflora, Commelena sp. and Cyperus sp. Pooled data revealed that, application of pyrazosulfuron ethyl $10 \% \mathrm{WP}$ at $20 \mathrm{~g}$ a.i./ha as pre-emergent herbicide followed by the application of Bispyribac sodium $10 \%$ SC @ $250 \mathrm{ml} / \mathrm{ha}$ at 20 to 25 days after sowing as post-emergent herbicide in direct seeded rice was most effective in controlling of grasses, broad leaf weeds and sedges and increased the rice grain yield $(5583 \mathrm{~kg} / \mathrm{ha})$ without any phytotoxic effect and which was onpar with the application of Pendimethalin 30 EC @ $1 \mathrm{~kg}$ a.i. /ha as pre-emergent herbicide followed by one hand weeding at 30 days after sowing and weed free check. Therefore, the application of pre emergent herbicides followed by the post emergent herbicide application can reduce the weed problem in direct seeded rice. .
\end{abstract}

Keywords: Grain yield, Weed control efficiency, Weed dry weight, Weeds

\section{INTRODUCTION}

Agriculture has been a forefront agenda at national and international level for food security and management of natural resources. Cereals are the most important part of our diet throughout the world and thus, play a major role in our food security. Among cereals, rice has been staple food for more than 60 per cent of the world population, providing energy for about $40 \%$ of the world population where every third person on earth consumes rice every day in one form or other. Therefore, paddy (Oryza sativa L.) is an important crop which is extensively grown in tropical and subtropical regions of the world. It is cultivated in area of 44.0 million hectares with an annual production of 104.3 million tons in India. Its production has been found to be distributed as 91.5 million tons in kharif and 12.8 million tons in rabi season. However, its productivity in India is very low $\left(2.37 \mathrm{t} \mathrm{ha}^{-1}\right)$ as compared to other rice growing countries like Japan $\left(6.35 \mathrm{t} \mathrm{ha}^{-1}\right)$, Australia $\left(6.22 \mathrm{tha}^{-1}\right)$, Spain $\left(6.16 \mathrm{t} \mathrm{ha}^{-1}\right)$, Egypt $\left(5.0 \mathrm{t} \mathrm{ha}^{-1}\right)$ and China (5.2 $\mathrm{t} \mathrm{ha}^{-1}$ ) (Annonymous, 2015).

Weeds are the major cause of yield reduction in rice. Hand weeding is the traditional weed control measure and still being the most popular in rice. However, due to high labour cost, non-availability of labour and huge time requirement for manual weeding, farmers are inevitable to go for other alternative measures like chemical weed control. Many herbicides are being used successfully for weed control in transplanted rice as pre -emergence spray. New herbicides are available in the market and use of herbicides of different composition is desirable to reduce the problem of residue buildup, shift in weed problem (Rajkhowa et al., 2006) and development of herbicide resistance in weeds (Rao, 1999, Saha et al., 2006). The recent trend of herbicide use is to find out an effective weed control measure by using low dose high efficiency herbicides which will not only reduce the total volume of herbicide use but also the application become easier and economical (Pal and Banerjee, 2007). The herbicide pyrazosulfuron ethyl $10 \%$ WP has both foliar and soil activity (Rajkhowa et al., 2006). It is generally recommended as a pre-emergence herbicide in transplanted rice (Angiras and Kumar, 2005). Studies on bioefficacy and phytotoxicity of pyrazosulfuron ethyl 10 $\% \mathrm{WP}$ and Pendimethalin $30 \mathrm{EC}$ for pre-emergence weed control and Bispyribac sodium $10 \%$ SC, Penoxsulam $24 \%$ SC, Metsulfuron methyl $10 \%+$ chlorimuron $10 \%$ and Azimsulfuron $50 \mathrm{DF}$ in direct seeded rice are scanty. The present experiment was therefore undertaken to study the bio-efficacy and phytotoxicity of pre-emergent followed by post emergent herbicides for control of major weeds in direct seeded rice. 


\section{MATERIALS AND METHODS}

An experiment was conducted during Kharif 2014 and 2015 at Agricultural Research Station, Dhadesugur, University of Agricultural Sciences (UAS), Raichur, Karnataka, India, (situated at 15.6' N latitude and 76.8' E longitude with an altitude of $358 \mathrm{~m}$ above mean sea level). The soil was deep black clay in texture having a $\mathrm{pH}$ of 8.1 , organic Carbon $0.21 \%$, total $\mathrm{N} 160 \mathrm{~kg} / \mathrm{ha}$, available P $26.0 \mathrm{~kg} / \mathrm{ha}$ and available $\mathrm{K} 486 \mathrm{~kg} / \mathrm{ha}$. The experiment was laid out in a randomized block design with ten treatments, viz. $\mathrm{T}_{1}$ - Pendimethalin30 EC @ 1 $\mathrm{kg}$ a.i. /ha followed by Bispyribac sodium 10\% SC @ $250 \mathrm{ml} / \mathrm{ha}, \mathrm{T}_{2}$ - Pendimethalin $30 \mathrm{EC} @ 1 \mathrm{~kg}$ a.i. /ha followed by 2,4 D sodium salt80 WP @600 g a.i./ha, T3-Pendimethalin30 EC @ $1 \mathrm{~kg}$ a.i. /ha followed by Azimsulfuron 50 DF @ $17.5 \mathrm{~g}$ a.i /ha, T4- Pendimethalin30 EC@1 kg a.i. /ha followed by Metsulfuron methyl 10\% + chlorimuron 10\%@4 g a.i. /ha, $\mathrm{T}_{5^{-}}$ Pyrazosulfuron ethyl 10 WP@20 g a.i. /ha followed by Metsulfuron methyl 10\%+chlorimuron 10\%@4 g a.i. /ha, $\mathrm{T}_{6}$ - Oxodiagil 80\%@100 g a.i. /ha followed by Penoxsulam 24 \% SC@25 g a.i./ha, T Pyrazosulfuron ethyl10 WP@20 g a.i. /ha followed by Bispyribac sodium $10 \% \mathrm{SC} @ 250 \mathrm{ml} / \mathrm{ha}, \mathrm{T}_{8^{-}}$ Pendimethalin30 EC @ 1 kg a.i. /ha followed by one hand weeding at 30 days after sowing $\mathrm{T}_{9^{-}}$weed free check (weeding at 15 and 30 days after sowing) and $\mathrm{T}_{10^{-}}$weedy check (Untreated check) and replicated thrice. Pre-emergent herbicides were applied with in three days after sowing and post emergent herbicides were applied at 20-25 days after sowing as per the treatment. The rice variety used was Gangavathi sona of 135 days duration. The crop was sown during $1 \mathrm{st}$ week of July in both the years. The herbicides were sprayed with the spray volume of 500 1/ha using knap- sack sprayer with flat fan nozzle. An area was selected randomly at three spots by making a quadrat of 0.25 $\mathrm{m}^{2}$. Weed species were counted from that area and density was expressed in number per square meter. The collected weeds were first sun-dried and then kept in an electric oven at $70{ }^{\circ} \mathrm{C}$ till the weight became constant and weed dry weight was expressed as $\mathrm{g} / \mathrm{m}^{2}$. As wide variation existed in data, number and biomass of weeds were transformed through square-root method before analysis of variance. Comparison of treatment means for significance at five per cent level was done using the critical differences as suggested by Gomez and Gomez (1984). Weed control efficiency (WCE) was worked out using the formula as suggested by Mani et al., 1973 and Gill and Vijayakumar, 1969. In direct seeded rice, five plants were randomly selected in each plot of each replication and were tagged for the purpose of recording observations on growth parameters viz., plant height and number of productive tillers per hill at harvest. Yield parameters viz., grain and straw yield. Similarly, rice from each net plot in each replication was harvested and dried. The grains after threshing were weighed and recorded as grain yield per net plot. Further, this net plot grain yield was converted to grain yield per hectare.

\section{RESULTS AND DISCUSSION}

Weed density and weed control efficiency: In the experimental plots, the dominant weeds were Echinochloa sp, Panicum repens, Cynodon doctylon, Leptochloa chinensis, Bracharia sp. Ludwigia parviflora, Commelena sp. All the herbicides (both pre and post emergent) showed effective control of all categories of dominant weeds resulting in less weed numbers per square meter, weed dry matter ( $g$ ) and higher weed

Table 1. Effect of weed control treatments on weed counts $\left(\mathrm{No} . / \mathrm{m}^{2}\right)$ in direct seeded rice at 15 days after sowing.

\begin{tabular}{|c|c|c|c|c|c|c|c|c|c|c|c|}
\hline \multirow{3}{*}{ Treatment } & \multicolumn{11}{|c|}{ Weed population (Count $\left./ \mathrm{m}^{2}\right)$} \\
\hline & \multicolumn{3}{|c|}{ Grasses $($ No./m²) } & \multicolumn{3}{|c|}{ Broad leaf weeds $\left(\right.$ No. $\left./ \mathbf{m}^{2}\right)$} & \multicolumn{3}{|c|}{ Sedges $($ No./m²) } & \multirow{2}{*}{$\begin{array}{c}\begin{array}{c}\text { Total weed } \\
\text { dry weight }(\mathrm{g} / \\
\left.\mathbf{m}^{2}\right)\end{array} \\
\text { Pooled } \\
\end{array}$} & \multirow{2}{*}{$\begin{array}{c}\begin{array}{c}\text { Weed con- } \\
\text { trol efficien- } \\
\text { cy }(\%)\end{array} \\
\text { Pooled }\end{array}$} \\
\hline & 2014 & 2015 & Pooled & 2014 & 2015 & Pooled & 2014 & 2015 & Pooled & & \\
\hline $\begin{array}{l}\mathbf{T}_{\mathbf{1}} \text { Pendimethalin fb Bispyri- } \\
\text { bac sodium }\end{array}$ & $\begin{array}{c}2.41 \\
(4.80)\end{array}$ & $\begin{array}{c}2.41 \\
(4.82)\end{array}$ & $\begin{array}{c}2.41 \\
(4.81)\end{array}$ & $\begin{array}{c}2.12 \\
(3.50)\end{array}$ & $\begin{array}{c}2.13 \\
(3.52)\end{array}$ & $\begin{array}{c}2.12 \\
(3.51)\end{array}$ & $\begin{array}{c}1.84 \\
(2.40)\end{array}$ & $\begin{array}{c}1.85 \\
(2.42)\end{array}$ & $\begin{array}{c}1.85 \\
(2.41)\end{array}$ & $\begin{array}{c}3.42 \\
(10.7)\end{array}$ & 57.0 \\
\hline $\begin{array}{l}\mathbf{T}_{2:} \text { Pendimethalin fb } 2,4 \mathrm{D} \\
\text { sodium salt }\end{array}$ & $\begin{array}{c}2.43 \\
(4.90)\end{array}$ & $\begin{array}{c}2.43 \\
(4.91)\end{array}$ & $\begin{array}{c}2.43 \\
(4.91)\end{array}$ & $\begin{array}{c}2.15 \\
(3.62)\end{array}$ & $\begin{array}{l}2.15 \\
(3.64)\end{array}$ & $\begin{array}{l}2.15 \\
(3.63)\end{array}$ & $\begin{array}{l}1.90 \\
(2.61)\end{array}$ & $\begin{array}{l}1.93 \\
(2.71)\end{array}$ & $\begin{array}{l}1.91 \\
(2.66)\end{array}$ & $\begin{array}{c}3.49 \\
(11.2)\end{array}$ & 55.2 \\
\hline $\begin{array}{l}\mathbf{T}_{3:} \quad \text { Pendimethalin } \mathrm{fb} \\
\text { Azimsulfuron }\end{array}$ & $\begin{array}{l}2.43 \\
(4.92)\end{array}$ & $\begin{array}{c}2.44 \\
(4.94)\end{array}$ & $\begin{array}{c}2.44 \\
(4.93)\end{array}$ & $\begin{array}{l}2.17 \\
(3.71)\end{array}$ & $\begin{array}{l}2.17 \\
(3.73)\end{array}$ & $\begin{array}{l}2.17 \\
(3.72)\end{array}$ & $\begin{array}{l}1.93 \\
(2.71)\end{array}$ & $\begin{array}{l}1.96 \\
(2.84)\end{array}$ & $\begin{array}{c}1.94 \\
(2.78)\end{array}$ & $\begin{array}{l}3.52 \\
(11.4)\end{array}$ & 54.3 \\
\hline $\begin{array}{l}\mathbf{T}_{\mathbf{4}} \text { Pendimethalin fb Metsul- } \\
\text { furon methyl }\end{array}$ & $\begin{array}{l}2.35 \\
(4.50)\end{array}$ & $\begin{array}{l}2.35 \\
(4.52)\end{array}$ & $\begin{array}{l}2.35 \\
(4.51)\end{array}$ & $\begin{array}{l}2.17 \\
(3.72)\end{array}$ & $\begin{array}{l}2.18 \\
(3.75)\end{array}$ & $\begin{array}{l}2.18 \\
(3.74)\end{array}$ & $\begin{array}{l}1.93 \\
(2.74)\end{array}$ & $\begin{array}{l}1.96 \\
(2.83)\end{array}$ & $\begin{array}{l}1.95 \\
(2.79)\end{array}$ & $\begin{array}{l}3.47 \\
(11.0)\end{array}$ & 55.8 \\
\hline 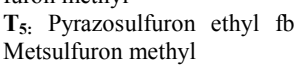 & $\begin{array}{c}2.29 \\
(4.26)\end{array}$ & $\begin{array}{c}2.30 \\
(4.28)\end{array}$ & $\begin{array}{c}2.30 \\
(4.27)\end{array}$ & $\begin{array}{l}2.20 \\
(3.86)\end{array}$ & $\begin{array}{c}2.08 \\
(3.32)\end{array}$ & $\begin{array}{c}2.14 \\
(3.59)\end{array}$ & $\begin{array}{l}1.77 \\
(2.12)\end{array}$ & $\begin{array}{l}1.80 \\
(2.24)\end{array}$ & $\begin{array}{l}1.78 \\
(2.18)\end{array}$ & $\begin{array}{c}3.32 \\
(10.0)\end{array}$ & 59.8 \\
\hline $\mathbf{T}_{6}$ : Oxodiagil fb Penoxsulam & $\begin{array}{c}2.49 \\
(5.21)\end{array}$ & $\begin{array}{l}2.50 \\
(5.24)\end{array}$ & $\begin{array}{c}2.49 \\
(5.23)\end{array}$ & $\begin{array}{l}2.18 \\
(3.74)\end{array}$ & $\begin{array}{c}2.19 \\
(3.81)\end{array}$ & $\begin{array}{c}2.19 \\
(3.78)\end{array}$ & $\begin{array}{l}1.98 \\
(2.94)\end{array}$ & $\begin{array}{l}1.99 \\
(2.95)\end{array}$ & $\begin{array}{l}1.99 \\
(2.95)\end{array}$ & $\begin{array}{l}3.60 \\
(11.9)\end{array}$ & 52.2 \\
\hline $\begin{array}{l}\text { T: }_{7:} \text { Pyrazosulfuron ethyl fb } \\
\text { Bispyribac sodium }\end{array}$ & $\begin{array}{l}2.29 \\
(4.25)\end{array}$ & $\begin{array}{l}2.29 \\
(4.26)\end{array}$ & $\begin{array}{l}2.29 \\
(4.26)\end{array}$ & $\begin{array}{l}2.06 \\
(3.24)\end{array}$ & $\begin{array}{l}2.08 \\
(3.34)\end{array}$ & $\begin{array}{l}2.07 \\
(3.29)\end{array}$ & $\begin{array}{l}1.80 \\
(2.24)\end{array}$ & $\begin{array}{l}1.83 \\
(2.34)\end{array}$ & $\begin{array}{c}1.81 \\
(2.29)\end{array}$ & $\begin{array}{l}3.29 \\
(9.84)\end{array}$ & 60.6 \\
\hline $\begin{array}{l}\mathbf{T}_{8:} \text { Pendimethalin }+\mathrm{HW} \text { at } \\
30 \text { DAP }\end{array}$ & $\begin{array}{c}2.43 \\
(4.91)\end{array}$ & $\begin{array}{c}2.43 \\
(4.92)\end{array}$ & $\begin{array}{c}2.43 \\
(4.92)\end{array}$ & $\begin{array}{c}2.15 \\
(3.62)\end{array}$ & $\begin{array}{c}2.17 \\
(3.71)\end{array}$ & $\begin{array}{l}2.16 \\
(3.67)\end{array}$ & $\begin{array}{l}1.98 \\
(2.92)\end{array}$ & $\begin{array}{c}1.99 \\
(2.95)\end{array}$ & $\begin{array}{l}1.98 \\
(2.94)\end{array}$ & $\begin{array}{c}3.54 \\
(11.5)\end{array}$ & 53.9 \\
\hline $\mathbf{T}_{9:}$ Weed free check & $\begin{array}{l}1.00 \\
(0.00)\end{array}$ & $\begin{array}{l}1.00 \\
(0.00)\end{array}$ & $\begin{array}{l}1.00 \\
(0.00)\end{array}$ & $\begin{array}{l}1.00 \\
(0.00)\end{array}$ & $\begin{array}{l}1.00 \\
(0.00)\end{array}$ & $\begin{array}{c}1.00 \\
(0.00)\end{array}$ & $\begin{array}{c}1.00 \\
(0.00)\end{array}$ & $\begin{array}{l}1.00 \\
(0.00)\end{array}$ & $\begin{array}{l}1.00 \\
(0.00)\end{array}$ & $\begin{array}{l}1.00 \\
(0.0)\end{array}$ & 100 \\
\hline $\mathbf{T}_{\mathbf{1 0}}$ : Weedy check & $\begin{array}{c}3.66 \\
(12.4)\end{array}$ & $\begin{array}{c}3.69 \\
(12.6)\end{array}$ & $\begin{array}{c}3.67 \\
(12.5)\end{array}$ & $\begin{array}{c}3.03 \\
(8.20)\end{array}$ & $\begin{array}{c}3.05 \\
(8.32)\end{array}$ & $\begin{array}{c}3.04 \\
(8.26)\end{array}$ & $\begin{array}{c}2.26 \\
(4.12)\end{array}$ & $\begin{array}{c}2.31 \\
(4.32)\end{array}$ & $\begin{array}{c}2.28 \\
(4.22)\end{array}$ & $\begin{array}{c}5.10 \\
(25.0)\end{array}$ & - \\
\hline$p=0.05$ & 0.35 & 0.26 & 0.38 & 0.52 & 0.56 & 0.54 & 0.65 & 0.42 & 0.51 & 1.82 & 3.65 \\
\hline
\end{tabular}

Figures in the parenthesis are square root transformed values (sq. root of $x+1$ ) fb: followed by 
Y.M. Ramesha et al. / J. Appl. \& Nat. Sci. 9 (3): 1866 -1870 (2017)

Table 2. Effect of weed control treatments on weed counts $\left(\mathrm{No} . / \mathrm{m}^{2}\right)$ in direct seeded rice at 30 days after sowing.

\begin{tabular}{|c|c|c|c|c|c|c|c|c|c|c|c|}
\hline \multirow{3}{*}{ Treatment } & \multicolumn{11}{|c|}{ Weed population (Count $/ \mathrm{m}^{2}$ ) } \\
\hline & \multicolumn{3}{|c|}{ Grasses (No./m²) } & \multicolumn{3}{|c|}{ Broad leaf weeds $\left(\mathrm{No} . / \mathrm{m}^{2}\right)$} & \multicolumn{3}{|c|}{ Sedges $\left(\mathbf{N o} . / \mathbf{m}^{2}\right)$} & \multirow{2}{*}{$\begin{array}{c}\begin{array}{c}\text { Total weed dry } \\
\text { weight }\left(\mathrm{g} / \mathbf{m}^{2}\right)\end{array} \\
\text { Pooled } \\
\end{array}$} & \multirow{2}{*}{$\begin{array}{c}\begin{array}{r}\text { Weed contro } \\
\text { efficiency }(\%\end{array} \\
\text { Pooled } \\
\end{array}$} \\
\hline & 2014 & 2015 & Pooled & 2014 & 2015 & Pooled & 2014 & 2015 & Pooled & & \\
\hline $\begin{array}{l}\mathbf{T}_{1:} \text { Pendimethalin fb } \\
\text { Bispyribac sodium }\end{array}$ & $\begin{array}{l}1.98 \\
(2.92)\end{array}$ & $\begin{array}{l}1.99 \\
(2.95)\end{array}$ & $\begin{array}{l}1.98 \\
(2.94)\end{array}$ & $\begin{array}{c}1.12 \\
(0.26)\end{array}$ & $\begin{array}{c}1.13 \\
(0.28)\end{array}$ & $\begin{array}{c}1.13 \\
(0.27)\end{array}$ & $\begin{array}{c}1.82 \\
(2.32)\end{array}$ & $\begin{array}{c}1.83 \\
(2.35)\end{array}$ & $\begin{array}{c}1.83 \\
(2.34)\end{array}$ & $\begin{array}{l}2.56 \\
(5.5)\end{array}$ & 87.2 \\
\hline $\begin{array}{l}\mathbf{T}_{2:} \text { Pendimethalin } \mathrm{fb} \\
2,4 \text { D sodium salt }\end{array}$ & $\begin{array}{l}2.05 \\
(3.21)\end{array}$ & $\begin{array}{c}2.14 \\
(3.56)\end{array}$ & $\begin{array}{c}2.09 \\
(3.39)\end{array}$ & $\begin{array}{l}1.49 \\
(1.21)\end{array}$ & $\begin{array}{l}1.50 \\
(1.26)\end{array}$ & $\begin{array}{l}1.49 \\
(1.24)\end{array}$ & $\begin{array}{l}1.91 \\
(2.65)\end{array}$ & $\begin{array}{c}1.92 \\
(2.69)\end{array}$ & $\begin{array}{l}1.92 \\
(2.67)\end{array}$ & $\begin{array}{l}2.88 \\
(7.3)\end{array}$ & 83.2 \\
\hline $\begin{array}{l}\mathbf{T}_{3} \text { : Pendimethalin } \mathrm{fb} \\
\text { Azimsulfuron }\end{array}$ & $\begin{array}{c}2.02 \\
(3.10)\end{array}$ & $\begin{array}{c}2.08 \\
(3.31)\end{array}$ & $\begin{array}{l}2.05 \\
(3.21)\end{array}$ & $\begin{array}{c}2.20 \\
(3.84)\end{array}$ & $\begin{array}{c}2.23 \\
(3.98)\end{array}$ & $\begin{array}{c}2.22 \\
(3.91)\end{array}$ & $\begin{array}{c}2.40 \\
(4.78)\end{array}$ & $\begin{array}{c}2.43 \\
(4.92)\end{array}$ & $\begin{array}{c}2.42 \\
(4.85)\end{array}$ & $\begin{array}{c}3.60 \\
(12.0)\end{array}$ & 72.5 \\
\hline $\begin{array}{l}\mathbf{T}_{4:} \text { Pendimethalin fb } \\
\text { Metsulfuron methyl }\end{array}$ & $\begin{array}{l}2.07 \\
(3.28)\end{array}$ & $\begin{array}{c}2.08 \\
(3.31)\end{array}$ & $\begin{array}{c}2.07 \\
(3.30)\end{array}$ & $\begin{array}{c}2.22 \\
(3.92)\end{array}$ & $\begin{array}{c}2.26 \\
(4.12)\end{array}$ & $\begin{array}{c}2.24 \\
(4.02)\end{array}$ & $\begin{array}{c}2.42 \\
(4.85)\end{array}$ & $\begin{array}{c}2.43 \\
(4.89)\end{array}$ & $\begin{array}{c}2.42 \\
(4.87)\end{array}$ & $\begin{array}{c}3.63 \\
(12.2)\end{array}$ & 72.0 \\
\hline $\begin{array}{l}\mathbf{T}_{5:} \text { Pyrazosulfuron } \\
\text { ethyl fb Metsulfuron } \\
\text { methyl }\end{array}$ & $\begin{array}{c}2.06 \\
(3.25)\end{array}$ & $\begin{array}{c}2.14 \\
(3.56)\end{array}$ & $\begin{array}{c}2.10 \\
(3.41)\end{array}$ & $\begin{array}{c}2.21 \\
(3.89)\end{array}$ & $\begin{array}{c}2.22 \\
(3.92)\end{array}$ & $\begin{array}{c}2.21 \\
(3.91)\end{array}$ & $\begin{array}{c}2.42 \\
(4.86)\end{array}$ & $\begin{array}{c}2.43 \\
(4.92)\end{array}$ & $\begin{array}{c}2.43 \\
(4.89)\end{array}$ & $\begin{array}{c}3.63 \\
(12.2)\end{array}$ & 71.9 \\
\hline $\begin{array}{l}\text { T}_{6:} \text { Oxodiagil } \mathrm{fb} \\
\text { Penoxsulam }\end{array}$ & $\begin{array}{c}2.07 \\
(3.29)\end{array}$ & $\begin{array}{l}2.08 \\
(3.32)\end{array}$ & $\begin{array}{l}2.07 \\
(3.31)\end{array}$ & $\begin{array}{c}2.20 \\
(3.86)\end{array}$ & $\begin{array}{c}2.21 \\
(3.89)\end{array}$ & $\begin{array}{c}2.21 \\
(3.88)\end{array}$ & $\begin{array}{c}2.49 \\
(5.21)\end{array}$ & $\begin{array}{c}2.50 \\
(5.24)\end{array}$ & $\begin{array}{c}2.49 \\
(5.23)\end{array}$ & $\begin{array}{c}3.66 \\
(12.4)\end{array}$ & 71.4 \\
\hline $\begin{array}{l}T_{7:} \text { Pyrazosulfuron } \\
\text { ethyl fb Bispyribac } \\
\text { sodium }\end{array}$ & $\begin{array}{c}1.97 \\
(2.89)\end{array}$ & $\begin{array}{c}1.98 \\
(2.91)\end{array}$ & $\begin{array}{c}1.97 \\
(2.90)\end{array}$ & $\begin{array}{c}1.11 \\
(0.24)\end{array}$ & $\begin{array}{c}1.12 \\
(0.25)\end{array}$ & $\begin{array}{c}1.12 \\
(0.25)\end{array}$ & $\begin{array}{c}1.50 \\
(1.26)\end{array}$ & $\begin{array}{c}1.51 \\
(1.29)\end{array}$ & $\begin{array}{c}1.51 \\
(1.28)\end{array}$ & $\begin{array}{c}2.33 \\
(4.42)\end{array}$ & 89.8 \\
\hline $\begin{array}{l}\mathbf{T}_{8:} \text { Pendimethalin }+ \\
\text { HW at } 30 \text { DAP }\end{array}$ & $\begin{array}{l}1.00 \\
(0.00)\end{array}$ & $\begin{array}{c}1.00 \\
(0.00)\end{array}$ & $\begin{array}{l}1.00 \\
(0.00)\end{array}$ & $\begin{array}{l}1.00 \\
(0.00)\end{array}$ & $\begin{array}{l}1.00 \\
(0.00)\end{array}$ & $\begin{array}{l}1.00 \\
(0.00)\end{array}$ & $\begin{array}{c}1.00 \\
(0.00)\end{array}$ & $\begin{array}{c}1.00 \\
(0.00)\end{array}$ & $\begin{array}{l}1.00 \\
(0.00)\end{array}$ & $\begin{array}{l}1.00 \\
(0.0)\end{array}$ & 100 \\
\hline $\mathbf{T}_{\mathbf{9}}$ : Weed free check & $\begin{array}{l}1.00 \\
(0.00)\end{array}$ & $\begin{array}{l}1.00 \\
(0.00)\end{array}$ & $\begin{array}{l}1.00 \\
(0.00)\end{array}$ & $\begin{array}{l}1.00 \\
(0.00)\end{array}$ & $\begin{array}{l}1.00 \\
(0.00)\end{array}$ & $\begin{array}{l}1.00 \\
(0.00)\end{array}$ & $\begin{array}{l}1.00 \\
(0.00)\end{array}$ & $\begin{array}{l}1.00 \\
(0.00)\end{array}$ & $\begin{array}{l}1.00 \\
(0.00)\end{array}$ & $\begin{array}{l}1.00 \\
(0.0)\end{array}$ & 100 \\
\hline $\mathbf{T}_{\mathbf{1 0}}$ : Weedy check & $\begin{array}{c}4.40 \\
(18.4)\end{array}$ & $\begin{array}{c}4.49 \\
(19.2)\end{array}$ & $\begin{array}{c}4.45 \\
(18.8)\end{array}$ & $\begin{array}{c}4.05 \\
(15.4)\end{array}$ & $\begin{array}{c}4.15 \\
(16.2)\end{array}$ & $\begin{array}{c}4.10 \\
(15.8)\end{array}$ & $\begin{array}{c}3.08 \\
(8.48)\end{array}$ & $\begin{array}{c}3.20 \\
(9.25)\end{array}$ & $\begin{array}{c}3.14 \\
(8.87)\end{array}$ & $\begin{array}{c}6.67 \\
(43.5)\end{array}$ & \\
\hline$p=0.05$ & 0.38 & 0.32 & 0.69 & 0.52 & 0.24 & 0.52 & 0.63 & 0.54 & 0.58 & 3.21 & 6.72 \\
\hline
\end{tabular}

Figures in the parenthesis are square root transformed values (sq. root of $x+1$ ), fb: followed by

Table 3. Effect of weed control treatments on weed counts $\left(\mathrm{No} . / \mathrm{m}^{2}\right)$ in direct seeded rice at 60 days after sowing.

\begin{tabular}{|c|c|c|c|c|c|c|c|c|c|c|c|}
\hline \multirow{3}{*}{ Treatment } & \multicolumn{11}{|c|}{ Weed population (Count $/ \mathrm{m}^{2}$ ) } \\
\hline & \multicolumn{3}{|c|}{ Grasses $\left(\right.$ No. $\left./ \mathbf{m}^{2}\right)$} & \multicolumn{3}{|c|}{ Broad leaf weeds $\left(\mathrm{No} . \mathrm{m}^{2}\right)$} & \multicolumn{3}{|c|}{ Sedges $($ No./m²) } & \multirow{2}{*}{$\begin{array}{c}\begin{array}{c}\text { Total weed } \\
\text { dry weight }(\mathrm{g} / \\
\left.\mathbf{m}^{2}\right)\end{array} \\
\text { Pooled }\end{array}$} & \multirow{2}{*}{$\begin{array}{c}\begin{array}{c}\text { Weed con- } \\
\text { trol efficien- } \\
\text { cy }(\%)\end{array} \\
\text { Pooled }\end{array}$} \\
\hline & 2014 & 2015 & Pooled & 2014 & 2015 & Pooled & 2014 & 2015 & $\begin{array}{c}\text { Poole } \\
\text { d }\end{array}$ & & \\
\hline $\begin{array}{ll}\mathbf{T}_{1:} \quad \text { Pendimethalin } & \mathrm{fb} \\
\text { Bispyribac sodium } & \end{array}$ & $\begin{array}{c}2.03 \\
(3.12)\end{array}$ & $\begin{array}{c}2.06 \\
(3.25)\end{array}$ & $\begin{array}{c}2.05 \\
(3.19)\end{array}$ & $\begin{array}{c}1.52 \\
(1.32)\end{array}$ & $\begin{array}{c}1.53 \\
(1.35)\end{array}$ & $\begin{array}{c}1.53 \\
(1.34)\end{array}$ & $\begin{array}{c}2.03 \\
(3.12)\end{array}$ & $\begin{array}{c}2.06 \\
(3.25)\end{array}$ & $\begin{array}{c}2.05 \\
(3.19)\end{array}$ & $\begin{array}{l}2.95 \\
(7.7)\end{array}$ & 84.3 \\
\hline $\begin{array}{l}\mathbf{T}_{2:} \text { Pendimethalin } \mathrm{fb} 2,4 \mathrm{D} \\
\text { sodium salt }\end{array}$ & $\begin{array}{c}2.06 \\
(3.26)\end{array}$ & $\begin{array}{c}2.07 \\
(3.27)\end{array}$ & $\begin{array}{l}2.07 \\
(3.27)\end{array}$ & $\begin{array}{c}1.91 \\
(2.65)\end{array}$ & $\begin{array}{l}1.92 \\
(2.67)\end{array}$ & $\begin{array}{l}1.91 \\
(2.66)\end{array}$ & $\begin{array}{c}2.05 \\
(3.21)\end{array}$ & $\begin{array}{c}2.14 \\
(3.56)\end{array}$ & $\begin{array}{c}2.09 \\
(3.39)\end{array}$ & $\begin{array}{l}3.21 \\
(9.3)\end{array}$ & 81.0 \\
\hline $\begin{array}{l}\mathbf{T}_{3:} \quad \text { Pendimethalin } \mathrm{fb} \\
\text { Azimsulfuron }\end{array}$ & $\begin{array}{c}2.29 \\
(4.25)\end{array}$ & $\begin{array}{c}2.30 \\
(4.28)\end{array}$ & $\begin{array}{c}2.29 \\
(4.27)\end{array}$ & $\begin{array}{c}2.42 \\
(4.85)\end{array}$ & $\begin{array}{c}2.43 \\
(4.89)\end{array}$ & $\begin{array}{c}2.42 \\
(4.87)\end{array}$ & $\begin{array}{c}2.58 \\
(5.65)\end{array}$ & $\begin{array}{c}2.60 \\
(5.74)\end{array}$ & $\begin{array}{c}2.59 \\
(5.70)\end{array}$ & $\begin{array}{c}3.98 \\
(14.8)\end{array}$ & 69.7 \\
\hline $\begin{array}{l}\mathbf{T}_{\text {4: Pendimethalin fb Met- }} \\
\text { sulfuron methyl }\end{array}$ & $\begin{array}{c}2.36 \\
(4.56)\end{array}$ & $\begin{array}{c}2.36 \\
(4.57)\end{array}$ & $\begin{array}{c}2.36 \\
(4.57)\end{array}$ & $\begin{array}{c}2.38 \\
(4.65)\end{array}$ & $\begin{array}{c}2.39 \\
(4.69)\end{array}$ & $\begin{array}{c}2.38 \\
(4.67)\end{array}$ & $\begin{array}{c}2.49 \\
(5.21)\end{array}$ & $\begin{array}{c}2.51 \\
(5.32)\end{array}$ & $\begin{array}{c}2.50 \\
(5.27)\end{array}$ & $\begin{array}{c}3.94 \\
(14.5)\end{array}$ & 70.4 \\
\hline $\begin{array}{l}\mathbf{T}_{5:} \text { Pyrazosulfuron ethyl } \mathrm{fb} \\
\text { Metsulfuron methyl }\end{array}$ & $\begin{array}{c}2.28 \\
(4.21)\end{array}$ & $\begin{array}{c}2.29 \\
(4.23)\end{array}$ & $\begin{array}{c}2.28 \\
(4.22)\end{array}$ & $\begin{array}{c}2.29 \\
(4.26)\end{array}$ & $\begin{array}{c}2.30 \\
(4.28)\end{array}$ & $\begin{array}{c}2.30 \\
(4.27)\end{array}$ & $\begin{array}{c}2.54 \\
(5.45)\end{array}$ & $\begin{array}{c}2.55 \\
(5.48)\end{array}$ & $\begin{array}{c}2.54 \\
(5.47)\end{array}$ & $\begin{array}{c}3.87 \\
(13.9)\end{array}$ & 71.5 \\
\hline $\begin{array}{l}\mathbf{T}_{6:} \text { Oxodiagil fb Penoxsu- } \\
\text { lam }\end{array}$ & $\begin{array}{c}2.29 \\
(4.26)\end{array}$ & $\begin{array}{c}2.30 \\
(4.29)\end{array}$ & $\begin{array}{c}2.30 \\
(4.28)\end{array}$ & $\begin{array}{c}2.28 \\
(4.21)\end{array}$ & $\begin{array}{c}2.29 \\
(4.26)\end{array}$ & $\begin{array}{c}2.29 \\
(4.24)\end{array}$ & $\begin{array}{c}2.67 \\
(6.12)\end{array}$ & $\begin{array}{c}2.74 \\
(6.51)\end{array}$ & $\begin{array}{c}2.70 \\
(6.32)\end{array}$ & $\begin{array}{c}3.98 \\
(14.8)\end{array}$ & 69.7 \\
\hline $\begin{array}{l}\mathbf{T}_{7:} \text { Pyrazosulfuron ethyl } \mathrm{fb} \\
\text { Bispyribac sodium }\end{array}$ & $\begin{array}{c}2.06 \\
(3.25)\end{array}$ & $\begin{array}{c}2.06 \\
(3.26)\end{array}$ & $\begin{array}{c}2.06 \\
(3.26)\end{array}$ & $\begin{array}{c}1.46 \\
(1.12)\end{array}$ & $\begin{array}{c}1.47 \\
(1.15)\end{array}$ & $\begin{array}{c}1.46 \\
(1.14)\end{array}$ & $\begin{array}{c}1.50 \\
(1.25)\end{array}$ & $\begin{array}{c}1.51 \\
(1.29)\end{array}$ & $\begin{array}{c}1.51 \\
(1.27)\end{array}$ & $\begin{array}{c}2.58 \\
(5.66)\end{array}$ & 88.4 \\
\hline $\begin{array}{l}\mathbf{T}_{8:} \text { Pendimethalin }+\mathrm{HW} \text { at } \\
30 \text { DAP }\end{array}$ & $\begin{array}{c}2.02 \\
(3.10)\end{array}$ & $\begin{array}{c}2.05 \\
(3.21)\end{array}$ & $\begin{array}{c}2.04 \\
(3.16)\end{array}$ & $\begin{array}{c}1.50 \\
(1.26)\end{array}$ & $\begin{array}{c}1.51 \\
(1.27)\end{array}$ & $\begin{array}{c}1.50 \\
(1.27)\end{array}$ & $\begin{array}{c}1.87 \\
(2.50)\end{array}$ & $\begin{array}{c}1.87 \\
(2.51)\end{array}$ & $\begin{array}{c}1.87 \\
(2.51)\end{array}$ & $\begin{array}{l}2.82 \\
(6.9)\end{array}$ & 85.9 \\
\hline $\mathbf{T}_{\mathbf{9}}$ : Weed free check & $\begin{array}{l}1.00 \\
(0.00)\end{array}$ & $\begin{array}{l}1.00 \\
(0.00)\end{array}$ & $\begin{array}{l}1.00 \\
(0.00)\end{array}$ & $\begin{array}{c}1.00 \\
(0.00)\end{array}$ & $\begin{array}{l}1.00 \\
(0.00)\end{array}$ & $\begin{array}{c}1.00 \\
(0.00)\end{array}$ & $\begin{array}{c}1.00 \\
(0.00)\end{array}$ & $\begin{array}{c}1.00 \\
(0.00)\end{array}$ & $\begin{array}{c}1.00 \\
(0.00)\end{array}$ & $\begin{array}{l}1.00 \\
(0.0)\end{array}$ & 100 \\
\hline $\mathbf{T}_{\mathbf{1 0}}$ : Weedy check & $\begin{array}{c}4.72 \\
(21.3)\end{array}$ & $\begin{array}{c}4.85 \\
(22.5)\end{array}$ & $\begin{array}{c}4.79 \\
(21.9)\end{array}$ & $\begin{array}{c}4.31 \\
(17.6)\end{array}$ & $\begin{array}{c}4.37 \\
(18.1)\end{array}$ & $\begin{array}{c}4.34 \\
(17.8)\end{array}$ & $\begin{array}{c}3.20 \\
(9.21)\end{array}$ & $\begin{array}{c}3.20 \\
(9.26)\end{array}$ & $\begin{array}{c}3.20 \\
(9.24)\end{array}$ & $\begin{array}{c}7.07 \\
(49.0)\end{array}$ & \\
\hline $\mathrm{p}=0.05$ & 0.42 & 0.54 & 0.56 & 0.48 & 0.65 & 0.62 & 0.48 & 0.32 & 0.35 & 1.17 & 4.21 \\
\hline
\end{tabular}

Figures in the parenthesis are square root transformed values (sq. root of $x+1$ ), fb: followed by

control efficiency (\%) at 15, 30 and 60 days after sowing compared to untreated check (Tables 1-3). The number of dominant broad-leaved, grass and sedge weeds were gradually decreased with the application of pre emergent herbicides followed by the application of post emergent herbicides in all the observations (15, 30 and 60 DAS). Significantly better weed control was observed with the application of Pyrazosulfuron ethyl $10 \%$ WP @ $20 \mathrm{~g}$ a.i. /ha as pre-emergent herbicide followed by the application of Bispyribac sodium $10 \%$ SC@ $250 \mathrm{ml} /$ ha as post emergent herbicide and which was onpar with the application of Pendimethalin $30 \mathrm{EC}$ (a) $1 \mathrm{~kg}$ a.i. /ha as pre-emergent herbicide followed by hand weeding at 30 days after sowing and weed free check. Significantly lower weed biomass/numbers at 15 days after herbicide application was recorded with pyrazosulfuron ethyl $10 \% \mathrm{WP}$ at $20 \mathrm{~g}$ a.i./ha compared to pendimethalin 30\% EC@1 kg a.i./ha and Oxodiagil 80\%@100 g a.i. /ha. Angiras and Kumar (2005) also found that application of pyrazosulfuronethyl at $15 \mathrm{~g} / \mathrm{ha}$ mixed with sand at $150 \mathrm{~kg} / \mathrm{ha}$ was effective to control weeds in rice which resulted in significantly lower weed density and biomass without any phytotoxic effect on rice plant. Pyrazosulfuron-ethyl at 
Y.M. Ramesha et al. / J. Appl. \& Nat. Sci. 9 (3): 1866 -1870 (2017)

Table 4. Effect of weed control treatments on growth and yield parameters of direct seeded rice.

\begin{tabular}{|c|c|c|c|c|c|c|c|c|c|c|c|c|}
\hline \multirow{2}{*}{ Treatment } & \multicolumn{3}{|c|}{ Plant height $(\mathrm{cm})$} & \multicolumn{3}{|c|}{ Productive tillers $\left(\mathrm{No} . / \mathbf{m}^{2}\right)$} & \multicolumn{3}{|c|}{ Grain yield (kg/ha) } & \multicolumn{3}{|c|}{ Straw yield (kg/ha) } \\
\hline & 2014 & 2015 & Pooled & 2014 & 2015 & Pooled & 2014 & 2015 & Pooled & 2014 & 2015 & Pooled \\
\hline $\begin{array}{l}\mathbf{T}_{1} \text { : Pendimethalin fb Bispyri- } \\
\text { bac sodium }\end{array}$ & 101 & 104 & 103 & 202 & 205 & 204 & 5426 & 5526 & 5476 & 5842 & 5982 & 5912 \\
\hline $\begin{array}{l}\mathbf{T}_{2:} \text { Pendimethalin fb } 2,4 \text { D } \\
\text { sodium salt }\end{array}$ & 100 & 102 & 101 & 200 & 202 & 201 & 5010 & 5142 & 5076 & 5421 & 5521 & 5471 \\
\hline $\begin{array}{l}\mathbf{T}_{3} \text { : Pendimethalin fb Azimsul- } \\
\text { furon }\end{array}$ & 99.6 & 101 & 100.5 & 199 & 200 & 200 & 4625 & 4812 & 4719 & 5121 & 5124 & 5123 \\
\hline $\begin{array}{l}\mathbf{T}_{4} \text { : Pendimethalin fb Metsul- } \\
\text { furon methyl }\end{array}$ & 99.4 & 100 & 99.8 & 199 & 201 & 200 & 4765 & 4978 & 4872 & 5267 & 5365 & 5316 \\
\hline $\begin{array}{l}\mathbf{T}_{5:} \text { Pyrazosulfuron ethyl fb } \\
\text { Metsulfuron methyl }\end{array}$ & 98.5 & 99.5 & 99.0 & 197 & 198 & 198 & 4925 & 4989 & 4957 & 5265 & 5312 & 5289 \\
\hline $\mathbf{T}_{6:}$ Oxodiagil fb Penoxsulam & 98.0 & 99.4 & 98.7 & 196 & 197 & 197 & 4900 & 4952 & 4926 & 5214 & 5321 & 5268 \\
\hline $\begin{array}{l}\mathbf{T}_{7:} \text { Pyrazosulfuron ethyl fb } \\
\text { Bispyribac sodium }\end{array}$ & 104 & 105 & 105 & 208 & 212 & 210 & 5545 & 5621 & 5583 & 6051 & 6112 & 6082 \\
\hline $\begin{array}{l}\mathbf{T}_{8:} \text { Pendimethalin }+\mathrm{HW} \text { at } 30 \\
\text { DAP }\end{array}$ & 105 & 107 & 106 & 211 & 215 & 213 & 5624 & 5742 & 5683 & 6012 & 6124 & 6068 \\
\hline $\mathbf{T}_{9:}$ Weed free check & 110 & 113 & 111 & 220 & 221 & 220 & 5894 & 5924 & 5909 & 6254 & 6325 & 6290 \\
\hline $\mathbf{T}_{10:}$ Weedy check & 94.0 & 96.2 & 95.1 & 188 & 186 & 187 & 2980 & 3256 & 3118 & 2995 & 3312 & 3154 \\
\hline$p=0.05$ & 17.2 & 15.92 & 16.2 & 19.2 & 15.8 & 16.2 & 471.5 & 401.2 & 435.0 & 415.0 & 345.0 & 382.0 \\
\hline
\end{tabular}

fb- followed by

Table 5. Effect of weed control treatments on Economics of direct seeded rice (Pooled data).

\begin{tabular}{|c|c|c|c|c|c|c|c|c|c|c|c|}
\hline \multicolumn{5}{|c|}{ Treatment } & \multicolumn{2}{|c|}{$\begin{array}{l}\text { Cost of cultiva- } \\
\text { tion }(/ / \text { ha) }\end{array}$} & \multicolumn{2}{|c|}{$\begin{array}{l}\text { Gross returns ( / } \\
\text { ha) }\end{array}$} & $\begin{array}{l}\text { Net returns } \\
\text { (//ha) }\end{array}$ & \multicolumn{2}{|c|}{ B: C ratio } \\
\hline \multicolumn{5}{|c|}{$\mathbf{T}_{1:}$ Pendimethalin fb Bispyribac sodium } & \multicolumn{2}{|c|}{34480} & \multicolumn{2}{|c|}{115432} & 80952 & \multicolumn{2}{|c|}{3.35} \\
\hline \multicolumn{5}{|c|}{$\mathbf{T}_{2:}$ Pendimethalin $\mathrm{fb} 2,4 \mathrm{D}$ sodium salt } & \multicolumn{2}{|c|}{32860} & \multicolumn{2}{|l|}{106991} & 74131 & \multicolumn{2}{|c|}{3.26} \\
\hline \multicolumn{5}{|c|}{$\mathbf{T}_{3}$ : Pendimethalin fb Azimsulfuron } & \multicolumn{2}{|c|}{33355} & \multicolumn{2}{|l|}{99493} & 66137 & \multicolumn{2}{|c|}{2.98} \\
\hline \multirow{2}{*}{\multicolumn{5}{|c|}{$\begin{array}{l}\mathbf{T}_{4} \text { : Pendimethalin fb Metsulfuron methyl } \\
\mathbf{T}_{5} \text { : Pyrazosulfuron ethyl fb Metsulfuron methyl }\end{array}$}} & \multicolumn{2}{|c|}{32755} & \multicolumn{2}{|c|}{102746} & 69991 & \multicolumn{2}{|c|}{3.14} \\
\hline & & & & & \multicolumn{2}{|c|}{31910} & \multicolumn{2}{|c|}{104429} & 72519 & \multicolumn{2}{|c|}{3.27} \\
\hline \multicolumn{5}{|c|}{$\mathbf{T}_{6:}$ Oxodiagil fb Penoxsulam } & \multicolumn{2}{|c|}{34111} & \multicolumn{2}{|c|}{103788} & 69677 & \multicolumn{2}{|c|}{3.04} \\
\hline \multirow{2}{*}{\multicolumn{5}{|c|}{$\begin{array}{l}\mathbf{T}_{7:} \text { Pyrazosulfuron ethyl fb Bispyribac sodium } \\
\mathbf{T}_{8} \text {. Pendimethalin }+\mathrm{HW} \text { at } 30 \text { DAP }\end{array}$}} & \multicolumn{2}{|c|}{33635} & \multicolumn{2}{|l|}{117742} & 84107 & \multicolumn{2}{|c|}{3.50} \\
\hline & & & & & \multicolumn{2}{|c|}{35605} & \multicolumn{2}{|c|}{119728} & 84123 & \multicolumn{2}{|c|}{3.36} \\
\hline \multicolumn{5}{|c|}{$\mathbf{T}_{9}$ : Weed free check } & \multicolumn{2}{|c|}{37000} & 124470 & & 87470 & 3.36 & \\
\hline $\mathbf{T}_{10}$ & Veedy che & & & & & & 65514 & & 34514 & 2.11 & \\
\hline $\mathbf{p}=\mathbf{0}$. & & & & & $\mathbf{N}$ & & NA & & 6529 & 0.25 & \\
\hline Mtls & $\begin{array}{l}\begin{array}{l}\text { Pendime- } \\
\text { thalin }\end{array} \\
\end{array}$ & $\begin{array}{l}\text { Bispyribac } \\
\mathrm{Na}\end{array}$ & $\begin{array}{llll}2 & 4 & \mathrm{D} & \mathrm{Na} \\
\text { salt } & & \\
\end{array}$ & & & $\begin{array}{l}\text { Metsulfu- } \\
\text { ron }\end{array}$ & $\begin{array}{l}\text { Penoxusu- } \\
\text { lum }\end{array}$ & $\begin{array}{l}\begin{array}{l}\text { Pyrazosulfu- } \\
\text { ron }\end{array} \\
\end{array}$ & Top star & Grain & Straw \\
\hline $\begin{array}{l}\text { Rs/ } \\
\text { ha }\end{array}$ & 1605 & 1875 & 255 & 750 & & 150 & 2188 & 760 & 923 & Rs $20 / \mathrm{kg}$ & Rs. $1 / \mathrm{kg}$ \\
\hline
\end{tabular}

20 and $25 \mathrm{~g} /$ ha significantly reduced weed density and total weed biomass of Cyperus iria, Echinochloa colona etc. when applied at 3 to 10 days after transplanting (Chopra and Chopra 2003). There was no phyto-toxic effect was observed in direct seeded rice when applied pre and post emergent herbicides.

Further, application of Pyrazosulfuron ethyl $10 \% \mathrm{WP}$ (a) $20 \mathrm{~g}$ a.i. /ha as pre-emergent herbicide followed by the application of Bispyribac sodium 10\% SC @250 $\mathrm{ml} /$ ha as post emergent herbicide recorded significantly lower weed dry weight $\left(9.84,4.42\right.$ and $5.66 \mathrm{~g} / \mathrm{m}^{2}$ at 15,30 and 60 days after sowing, respectively) and which was onpar with the application of Pendimethalin $30 \%$ EC@ 1 kg a.i./ha as pre-emergent herbicide followed by hand weeding at 30 days after sowing $\left(11.5,0.00\right.$ and $2.51 \mathrm{~g} / \mathrm{m}^{2}$ at 15,30 and 60 days after sowing, respectively) and weed free check. The overall result showed that, Pyrazosulfuron ethyl 10\%WP @ $20 \mathrm{~g}$ a.i. /ha as pre-emergent herbicide followed by the application of Bispyribac sodium 10\% SC @ 250 ml/ ha as post emergent herbicide was comparatively more effective against broad-leaved, grassy and sedge weeds in direct seeded rice.
Similarly, Weed control efficiency (100\%) was higher in weed free check at all three dates of observation (15, 30 and 60 DAS) compared to other treatments. Application of Pyrazosulfuron ethyl 10\%WP @20 g a.i. / ha as pre-emergent herbicide followed by the application of Bispyribac sodium 10\% SC @250 ml/ha as post emergent herbicide recorded significantly higher weed control efficiency $(60.6,89.8$ and $88.4 \%$ at 15 , 30 and 60 days after sowing, respectively) and which was onpar with the application of Pendimethalin $30 \%$ EC @ $1 \mathrm{~kg}$ a.i./ha as pre-emergent herbicide followed by hand weeding at 30 days after sowing (53.9, 100 and $85.9 \%$ at 15,30 and 60 days after sowing, respectively).

Growth of direct seeded rice: Significantly taller plants and more number of productive tillers per hill were observed in weed free treatment $(111 \mathrm{~cm}$ and 220) and which was onpar with the application of Pyrazosulfuron ethyl $10 \% \mathrm{WP} @ 20 \mathrm{~g}$ a.i. /ha as preemergent herbicide followed by the application of Bispyribac sodium $10 \% \mathrm{SC} @ 250 \mathrm{ml} / \mathrm{ha}$ as post emergent herbicide (105 cm and 210) and the application of Pendimethalin 30 \% EC @ 1 kg a.i./ha as pre- 
emergent herbicide followed by hand weeding at 30 days after sowing (105 cm and 213) compared to other weed control treatments. Whereas, shorter plants and less number of productive tillers per hill were recorded in the weedy check (Table 4).

Grain and straw yield: Significantly higher grain and straw yield were recorded in weed free treatment (5909 and $6290 \mathrm{~kg} / \mathrm{ha}$, respectively) and which was onpar in the treatment with the application of Pyrazosulfuron ethyl 10\%WP@20 g a.i. /ha as pre-emergent herbicide followed by the application of Bispyribac sodium $10 \%$ SC@250 ml/ha as post emergent herbicide (5583 and $6082 \mathrm{~kg} / \mathrm{ha}$, respectively) and the application of Pendimethalin $30 \%$ EC @ $1 \mathrm{~kg}$ a.i./ha as preemergent herbicide followed by hand weeding at 30 days after sowing (5683 and $6068 \mathrm{~kg} / \mathrm{ha}$, respectively) compared to other weed control treatments (Table 4). Similar trend was recorded with respect to yield parameters of paddy. Pyrazosulfuron-ethyl at 20 and 25 $\mathrm{g} /$ ha provided grain yield statistically similar to weed free treatment (Chopra and Chopra, 2003, Pal and Banerjee, 2007). Whereas, lower grain and straw yield were recorded in weedy check plot. This was due to the higher infestation of weeds.

Economics of direct seeded rice: Application of Pyrazosulfuron ethyl 10\%WP @ $20 \mathrm{~g}$ a.i. /ha as preemergent herbicide followed by the application of Bispyribac sodium $10 \%$ SC @ $250 \mathrm{ml} / \mathrm{ha}$ as post emergent herbicide recorded significantly higher benefit cost ratio (3.50) and which was onpar with the application of Pendimethalin 30\% EC @ 1 kg a.i./ha as pre-emergent herbicide followed by hand weeding at 30 days after sowing (3.36) and weed free treatment (3.36) compared to other weed control treatments (Table 5). This might be due to higher grain yield. Application of pyrazosulfuron ethyl10\%WP@20 g a.i./ ha reduced grasses, broad leaf weeds and sedges in transplanted paddy (Chopra and Chopra, 2003). Whereas, significantly lower benefit cost ratio (2.11) was reported in weedy check plot. This might be due to the higher infestation (100\%) of weeds.

\section{Conclusion}

Results are concluded that, application of Pyrazosulfuron ethyl 10\%WP@20 g a.i. /ha as pre-emergent herbicide followed by the application of Bispyribac sodium $10 \%$ SC@250 ml/ha as post emergent herbicide or application of Pendimethalin 30\% EC @ 1 kg a.i./ha as pre-emergent herbicide followed by hand weeding at 30 days after sowing was effectively controlled the broad leaved, grasses and sedges and led to higher grain yield in direct seeded rice.

\section{REFERENCES}

Annonymous, (2015). Annual report 2014-15, Department of Agriculture and Cooperation Ministry Of Agriculture, Government of India Krishi Bhavan, New Delhi

Angiras, N.N. and Kumar, S. (2005). Efficacy of pyrazosulfuron-ethyl against weeds in rice nursery under mid hill conditions of Himachal Pradesh. Indian Journal of Weed Science, 37(3\&4):151-154

Chopra, N.K. and Chopra, N. (2003). Effect of doses and stages of application of pyrazosulfuron ethyl on weeds in transplanted rice. Indian Journal of Weed Science, 35 (1\&2): $42-46$

Gill, H.S. and Vijayakumar. (1969). Weed index-a new method for reporting weed control trial. Indian Journal of Agronomy, 14(1): 96-98.

Gomez, K.A. and Gomez, A.A. (1984). Statistical Procedures for Agricultural Research, John Wiley \& Sons. Inc., New York.

Mani, V.S., Pandita, M.L., Gautam, K.C. and Das, B. (1973). Weed killing chemicals in potato cultivation. Indian Farming, 23: 7-13

Pal, S. and Banerjee, H. (2007). Efficacy of penoxsulam against weeds in transplanted Kharif rice (Oryza sativa L.). Indian Journal of Weed Science, 39(3\&4):172-175

Rajkhowa, D.J., Borah, N., Barua, I.C. and Deka, N.C., (2006). Effect of pyrazosulfuron-ethyl on weeds and productivity of transplanted rice during rainy season. Indian Journal of Weed Science, 38:25-28

Rao, V.S. (1999). Principles of Weed Science, 2nd ed. Oxford \& IBH Publishing Co. Pvt. Ltd. pp. 277

Saha, M., Banerjee, H. and Pal, S. (2006). Relative efficacy of herbicides in wheat. Indian Journal of Weed Science 38(1\&2):127-128 